\title{
Changement institutionnel et contexte local : le cas de la Baie-James
}

\author{
Marie-France McSween et Emmanuel Raufflet \\ HEC Montréal
}

\section{Introduction}

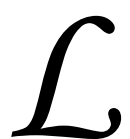
a collaboration entre les différentes organisations et entreprises d'une région ou d'un territoire représente un outil important pour le développement local. En effet, la mise en commun des forces, des habiletés et des ressources de chacun contient la promesse d'augmenter le potentiel de développement économique et social au niveau local. Cet esprit de collaboration revêt un caractère d'innovation particulièrement fort dans un contexte régional où les différentes communautés ont une longue tradition de travail à part.

Le 7 février 2002, à la suite de près de deux décennies d'affrontements, le gouvernement du Québec et le Grand Conseil des Cris signaient l'Entente concernant une nouvelle relation entre le gouvernement $d u$ Québec et le les Cris du Québec, dite «la Paix des Braves ». Cette entente de nation à nation repose sur un renforcement des relations politiques, économiques et sociales entre le Québec et les Cris, la coopération, le partenariat ainsi que le respect mutuel devant être au cœur de cette nouvelle relation ${ }^{1}$. Grâce à l'esprit de collaboration de la Paix des Braves, selon le gouvernement du Québec, « un essor économique et social sans précédent s'ouvre aux Cris et aux Québécois dans le Norddu-Québec $»^{2}$. Des emplois, des ressources financières ainsi que l'utilisation et le développement du territoire dans un souci de préoccupations communes sont des points importants de l'entente. En outre, la Paix des Braves s'inscrit en continuité de la Politique de développement du Nord-du-Québec de 2001 qui affirme le rôle important des entreprises - et en particulier des partenariats - dans le rapprochement entre communautés autochtones et non autochtones sur le territoire pour le développement local.

L'objet de cet article est d'examiner les effets de cette promotion de la collaboration et des partenariats entre entreprises jamésiennes (non autochtones de la Baie James) et cries auprès de deux communautés du territoire de la Baie-James, Radisson et Chisasibi, à partir d'une recherche de terrain réalisée en 2004 et 2005. Utilisant un cadre d'analyse qui évalue le degré d'appropriation par les acteurs locaux des valeurs et des capacités liées à la collaboration, nous documentons ici les dimensions locales de ce changement institutionnel.

Cet article comprend trois parties. Nous présentons d'abord le contexte géographique et historique récent de la Baie-James. Les relations entre les nations crie et québécoise autour des enjeux de l'utilisation des ressources naturelles depuis les années 1970 ont été caractérisées par de fortes tensions, des cycles d'affrontements et un niveau élevé de méfiance que la Paix des Braves de 2002 vise à dépasser. Ensuite, nous présentons nos résultats de recherche qui évaluent comment les acteurs locaux se représentent l'esprit de collaboration des grands discours au niveau provincial - par la Paix des Braves et la Politique de développement du Norddu-Québec - dans deux communautés de la région en 2004-2005. Pour cartographier comment ces acteurs se représentent cette collaboration afin de mieux développer leurs communautés, nous utilisons un cadre d'analyse provenant de la théorie institutionnelle. Greenwood et Hinings ${ }^{3}$ proposent qu'un changement institutionnel ne se réalisera que si les acteurs qui vivent ce changement sur le terrain adoptent les nou- 
velles valeurs de cette institution et s'ils ont les capacités pour l'action, c'est-à-dire les capacités d'opérer et de vivre ce changement. Nous évaluons donc dans cet article comment les acteurs locaux adoptent les valeurs et se donnent des capacités pour l'action. La conclusion propose un bref résumé de la situation et suggère une piste pour son amélioration.

\section{La Baie-James : un vaste territoire peu peuplé, terrain de confrontations entre nations}

Le territoire de la Baie-James, près de 350000 kilomètres carrés, se situe entre le $49^{\mathrm{e}}$ et le $55^{\mathrm{e}}$ parallèle, entre l'Abitibi-Témiscamingue et le Nunavik. A l'ouest, il est délimité par la frontière entre le Québec et l'Ontario, les rives de la baie James et de la baie d'Hudson; à l'est, principalement par la ligne de partage des eaux créée par les monts Otish. Sur ce territoire vivent environ 28950 personnes $^{4}$ réparties dans huit communautés cries et sept communautés jamésiennes. Les communautés cries sont Chisasibi, Wemindji, Eastmain, Waskaganish, Mistissini, Nemaska, Oujé-Bougoumou et Waswanipi. Une neuvième communauté crie, Whapmagoostui, se trouve au nord du territoire, au Nunavik. Dans les communautés jamésiennes, nous retrouvons quatre villes, Chapais, Chibougamau, Lebel-sur-Quevillon et Matagami, ainsi que trois localités, Radisson, Valcanton et Villebois. Ces sept communautés jamésiennes forment la municipalité de la Baie-James, souvent comparée à une municipalité régionale de comté (MRC). Le conseil municipal de la municipalité de la Baie-James est composé des maires et présidents de chaque ville et localité, en plus d'une huitième personne représentant le « territoire libre ».

Radisson se situe au nord du $53^{\mathrm{e}}$ parallèle, au bout de la route de la Baie-James. Ses plus proches voisines sont des communautés cries : Chisasibi $(125 \mathrm{~km})$, Wemindji $(202 \mathrm{~km})$ et Eastmain $(377 \mathrm{~km})$. Radisson comptait, au printemps 2001, une population d'environ 400 personnes, la très grande majorité francophone. Pour sa part, Chisasibi se situe aussi au nord du $53^{\mathrm{e}}$ parallèle. Elle se trouve à l'embouchure de la rivière La Grande, près des rives de la baie James. La communauté est reliée à la route de la Baie-James par une route faisant environ $100 \mathrm{~km}$. Avec 3500 personnes, elle est la communauté crie la plus importante en termes de population sur le territoire de la BaieJames $^{5}$. La majorité des Cris parlent le cri à la mai- son, mais peuvent aussi s'exprimer dans une autre langue qui, pour la majorité, est l'anglais.

Les Cris ont fait de cette région leur milieu de vie il y a environ 3500 ans : un territoire qu'ils nomment Eeyou Istchee, signifiant «terre crie » ou «territoire cri ». Peuple nomade, les familles cries parcourent depuis très longtemps ce territoire afin de subvenir à leurs besoins. C'est au début du $20^{\mathrm{e}}$ siècle que Fort George devint la première communauté semi-permanente crie du territoire de la Baie-James où quelques familles se sédentarisèrent. Tout d'abord poste de traite pour différentes compagnies, dont la Compagnie de la Baie d'Hudson, une école y vit le jour en 1907 ainsi qu'un premier hôpital en 1927. Dans les années 1980, avec le déménagement sur la terre ferme, la communauté prit le nom de Chisasibi.

\section{De 1970 à 1975 : une première période d'affrontements}

$\mathrm{Au}$ mois d'avril 1971, le premier ministre Robert Bourassa annonça, sans consultation préalable avec les Cris, le développement hydroélectrique de la rivière La Grande. En 1972, ces derniers commencèrent à revendiquer leurs droits sur le territoire. Ils demandèrent à la Cour supérieure du Québec une déclaration en nullité de la loi 50 qui porte sur le développement de la région de la Baie-James et qui a créé la Société de développement de la Baie-James (SDBJ). Selon les Cris, cette loi est inconstitutionnelle puisque jamais une entente n'avait été conclue entre eux et le gouvernement du Québec afin que ce dernier puisse développer le territoire. En effet, en 1898 et 1912, le Québec a reçu du gouvernement canadien le territoire de la Baie-James et le Nunavik, à condition qu'il conclue une entente avec les Cris et les Inuits pour que ceux-ci cèdent leurs droits sur le territoire, ce qui permettrait au Québec de jouir des ressources de ce même territoire. Le juge Malouf donna alors raison aux Cris et imposa une suspension des travaux le 15 novembre 1973. Par contre, cinq jours après la suspension des travaux de la Baie-James, la Cour d'appel du Québec renversa le jugement Malouf. Sans contester ce dernier, la Cour d'appel mentionnait des intérêts supérieurs aux droits des Autochtones ${ }^{6}$.

L'intérêt de quelque 2000 habitants de la province ne faisait pas le poids face aux intérêts des autres Québécois pour le développement du potentiel hydroélectrique. Par contre, les Cris obtinrent « des gouverne- 
ments du Québec et du Canada, contre l'abandon formellement exprimé de leurs droits au territoire et dans le territoire, l'assurance de tenir un rôle significatif auquel ils aspiraient dans la dynamique de la gestion et du développement du territoire $»^{7}$. Des négociations entre des représentants du gouvernement du Québec, du gouvernement du Canada et des Cris furent donc entreprises pour en arriver à une entente de principe le 15 novembre 1974. L'entente de principe de 1974 mena à la Convention de la Baie-James et du Nord québécois, signée un an plus tard, le 11 novembre 1975. Ainsi, plus de 60 ans après la loi de 1912, le Québec se conformait finalement à son obligation de conclure une entente avec les Cris et les Inuits, tel que le prescrit la loi. L'article 2.1 de la Convention affirme que : «En considération des droits et des avantages accordés aux présentes aux Cris de la Baie-James et aux Inuits du Québec, les Cris de la Baie-James et les Inuits du Québec cèdent, renoncent, abandonnent et transportent par les présentes tous leurs revendications, droits, titres et intérêts autochtones, quels qu'ils soient aux terres et dans les terres du Territoire et du Québec, et le Québec et le Canada acceptent cette cession $\gg{ }^{8}$.

La Convention de la Baie James et du Nord québécois repose sur deux grands principes. Le premier est de donner la chance à tous les Québécois de pouvoir bénéficier des ressources naturelles du territoire puisque ce dernier fait partie de la province de Québec. Le second vise à assurer la survie politique, sociale, culturelle et économique des peuples cri et inuit puisque celle-ci est essentielle au développement du territoire. Commença alors une période d'efforts de cohabitation dans la région. Les résidents de Fort George votèrent, lors d'un référendum, de quitter l'île afin de s'installer dans la nouvelle communauté de Chisasibi à 9 kilomètres en amont sur les rives de la rivière $\mathrm{La}$ Grande. Le déménagement eut lieu en 1981. En effet, l'aménagement du complexe hydroélectrique de la rivière La Grande allait faire augmenter le débit d'eau de la rivière, ce qui risquait de créer l'érosion des berges de leur île.

Parallèlement, Radisson, établi en 1974 pour loger une partie des travailleurs de la construction de la centrale La Grande-2, vit sa population augmenter jusqu'en 1978. Contrairement au campement des travailleurs, le village permit à ceux qui «montaient» pour plusieurs années, comme les cadres, d'amener leur famille avec eux. Le village offrait alors les ser- vices nécessaires tels qu'une école et un hôpital. Au plus fort des travaux, soit en 1978, 2500 personnes résidaient à Radisson. À la fin des travaux, tout comme le campement, le village devait être démoli et seul le complexe de résidences pour les travailleurs d'Hydro-Québec était censé demeurer. Par contre, une volonté d'habiter à Radisson se fit sentir au début des années 1980 et, en 1984, le village fut ouvert au public afin que tous ceux qui le souhaitaient puissent venir s'y installer.

La signature de la Convention a donc permis la poursuite, dans la paix, des travaux de la phase 1 du complexe La Grande entre 1973 et 1984, et des travaux correcteurs ont été entrepris afin de respecter la volonté des Cris sur le complexe La Grande.

\section{L'intérêt de quelque 2000 habitants de la province ne faisait pas le poids face aux intérêts des autres Québécois pour le développement du potentiel hydroélectrique.}

\section{Une deuxième période d'affrontements}

Cependant, du milieu des années 1980 à 2001, s'amorça une deuxième période d'affrontements. En effet, il apparaît qu'au fil des ans, le gouvernement du Québec ne respecta pas dans tout son esprit la Convention de la Baie James et ses conventions subséquentes, et ne respecta pas toutes ses promesses. Cette absence de respect de la Convention serait expliquée par plusieurs facteurs. Un premier a trait à « l'absence de conscientisation de l'appareil gouvernemental québécois à la réalité autochtone $»$. Un deuxième est davantage lié à l'organisation de l'appareil administratif québécois. Aucune structure ne fut mise en place du côté du gouvernement québécois pour s'assurer de la coordination des activités liées à la Convention. Il ne put compter que sur ses ministères ou sur ses entreprises publiques peu coordonnés entre eux car dépourvus d'une entité chargée de la mise en œuvre.

Cette faille initiale d'organisation conduisit à la détérioration des relations avec les Cris qui eurent recours aux tribunaux pour plusieurs différends. Cette détérioration des relations mena à l'opposition croissante des Cris aux projets du gouvernement du Québec sur le territoire. 
De son côté, Radisson reçut le titre de « localité de la municipalité de la Baie-James » en 1994. Évidemment, selon les périodes des chantiers du complexe hydroélectrique, Radisson vit sa population chuter rapidement de 1500 personnes en 1990 à 500 en 1995. Afin de pallier le déclin de la population enclenché par la fin des travaux de la phase 2 du complexe La Grande ${ }^{10}$, Radisson se dota d'un plan de développement stratégique (1995-2000) qui visait à relancer la localité ${ }^{11}$. D'abord, stabiliser la population, donner la possibilité d'acheter un fonds de terre, avoir de bons services pour assurer une qualité de vie, élargir l'offre de produits touristiques, etc. Ensuite, demander à Hydro-Québec d'inciter ses employés à s'établir à Radisson plutôt que de faire des allersretours en avion. Enfin, maximiser les relations avec les communautés autochtones en apprenant la langue et la culture crie, en multipliant les échanges entre élus, gens d'affaires et organismes des loisirs et socioculturels de Radisson et de Chisasibi et en favorisant le maillage d'entreprises cries et jamésiennes. Dès 1996, les résidents pouvaient acheter leur terrain, ce qui donna un plus grand sentiment d'appartenance à la population.

\section{Le développement du territoire peut se faire de façon plus efficace si les entreprises cries et jamésiennes créent des partenariats entre elles.}

\section{Depuis 2001 : des politiques de promotion de collaboration et de partenariats d'affaires}

En octobre 2001, Ted Moses, Chef du Grand Conseil des Cris, et Bernard Landry, Premier ministre du Québec, déposaient une entente de principe qui aboutit à la signature de la Paix des Braves le 7 février 2002. Avec cette signature, le Grand Conseil des Cris et le gouvernement du Québec démontraient une volonté commune de poursuivre en collaboration le développement du territoire de la Baie-James par l'essor minier, forestier et hydroélectrique, ainsi que l'épanouissement de la nation crie, et tenaient à ce que cette dernière ait une plus grande prise en charge de son propre développement. Afin de s'assurer de sa mise en œuvre et d'éviter les erreurs du passé, un comité de liaison permanent fut mis en place qui représentait un lieu d'échange et de coordination visant à renforcer les relations entre les deux nations, de s'assurer du suivi ainsi que de trouver des solutions aux différents problèmes qui pouvaient survenir.

Parallèlement, en 2001, dans un même esprit de promotion de collaboration, le gouvernement du Québec déposait sa Politique de développement du Nord-duQuébec qui mentionne les trois nations de cette région, soit les Québécois, les Cris et les Inuits. Dans le cas de la Baie-James, la Politique de développement souligne, comme il a déjà été mentionné, que le développement du territoire peut se faire de façon plus efficace si les entreprises cries et jamésiennes créent des partenariats entre elles. En résumé, tant la Paix des Braves que la Politique de développement du Nord-du-Québec placent l'esprit de collaboration entre les Jamésiens et les Cris au cœur des politiques provinciales ou de "nation à nation » afin de développer le territoire.

\section{Cadre d'analyse et résultats}

\section{L'esprit de collaboration au niveau local}

Pour examiner dans quelle mesure cette pensée provinciale de promotion de la collaboration se répercute localement, nous utilisons un cadre conceptuel simple issu des recherches sur le changement institutionnel. Greenwood et Hinings ${ }^{12}$ estiment qu'un changement au niveau local à partir du niveau provincial se réalisera en fonction de deux conditions. La première a trait à l'adoption par les acteurs sur le terrain des nouvelles valeurs de ce changement. La seconde porte sur leurs capacités pour l'action, c'est-à-dire les capacités d'opérer et de vivre ce changement.

Selon ces auteurs, il existe quatre niveaux d'engagement face à de nouvelles valeurs promues par un changement institutionnel. Les acteurs peuvent donc épouser une des quatre formes d'engagement aux valeurs :

- le statu quo, dans lequel les acteurs rejettent totalement les nouvelles valeurs et gardent les valeurs préexistantes au changement institutionnel;

- l'engagement indifférent, où les acteurs ne s'opposent ni ne s'engagent aux nouvelles valeurs;

- l'engagement compétitif, où certains acteurs soutiennent le modèle de valeurs préexistantes tandis 
que d'autres préfèrent une alternative prenant racine dans le nouveau contexte institutionnel;

- l'engagement réformateur, dans lequel tous les acteurs s'approprient les valeurs du nouveau contexte institutionnel et s'opposent aux valeurs préexistantes.

Greenwood et Hinings affirment que l'engagement des acteurs doit être compétitif ou réformateur afin que le changement se réalise. Le changement requiert donc qu'une masse critique d'acteurs soutienne ces nouvelles valeurs. Évidemment, plus l'engagement sera réformateur, plus le changement se fera d'une façon radicale.

D'autre part, ils distinguent trois capacités pour l'action :

- La compréhension : bien comprendre le nouveau contexte institutionnel. Afin d'effectuer un changement dans la dynamique locale en fonction d'un changement au niveau provincial, les acteurs doivent comprendre le changement au niveau provincial. Par exemple, les acteurs sur les territoires de la Baie-James doivent comprendre pourquoi un nouvel esprit de collaboration s'installe au niveau provincial et voir ses avantages avant de vouloir l'appliquer dans son milieu.

- Le fonctionnement : avoir les habiletés pour fonctionner dans ce nouveau contexte institutionnel. Si les acteurs n'ont pas les habiletés pour fonctionner dans ce nouveau contexte institutionnel, le changement ne s'exercera pas au niveau local. Par exemple, dans un contexte de collaboration, la différence de culture entre les Cris et les Jamésiens peut apporter des frustrations et des problèmes dans les partenariats entre eux. S'ils n'ont pas les moyens de dépasser cette barrière culturelle, le changement au niveau local s'exercera difficilement ou pas du tout.

- Le changement : avoir les habiletés pour gérer le changement vers le nouveau contexte institutionnel. Encore une fois, si les acteurs n'ont pas les habiletés pour gérer le changement vers le nouveau contexte institutionnel, c'est-à-dire vers la collaboration, le changement ne pourra se faire. Par exemple, si les acteurs dans la région de Chisasibi et de Radisson ne se dotent pas d'outils, tel un organisme pouvant créer des ponts entre des entrepreneurs cris et jamésiens pour rapprocher les deux communautés, la gestion du changement en sera plus difficile et le changement ne se verra pas dans la dynamique locale.

Les deux hypothèses des auteurs à ce sujet sont : 1) qu'il n'y aura pas de changement radical au niveau de l'organisation (ici de la dynamique de développement local) sans des capacités pour l'action assez fortes combinées à un engagement réformateur ou compétitif aux nouvelles valeurs; et 2) qu'une grande capacité pour l'action permettra un changement plus révolutionnaire. Une combinaison de l'engagement des acteurs aux nouvelles valeurs et des capacités pour l'action de ceux-ci viendra donc aider ou nuire au changement.

Nous nous sommes concentrés sur les deux communautés de Chisasibi et Radisson. Le changement de discours au niveau provincial est le changement institutionnel étudié. Ce discours est maintenant davantage axé sur la collaboration entre les deux nations pour développer le territoire de la Baie-James, ce qui contraste avec ces années de différends entre les Cris et le gouvernement du Québec. Cette collaboration est aussi souhaitée au niveau local afin de créer un développement plutôt endogène, ou intercommunautaire, sur le territoire de la Baie-James, par les Cris et les Jamésiens. Un nouveau contexte institutionnel devrait insuffler des valeurs de collaboration au niveau local et les aider à se donner des capacités pour l'action afin d'obtenir un changement dans la dynamique de développement local à Chisasibi et à Radisson, pour le cas qui nous intéresse.

\section{Le changement requiert qu'une masse critique d'acteurs soutienne ces nouvelles valeurs.}

Nous présentons ici les résultats de notre étude de terrain réalisée en 2004 et 2005. En plus d'observations, treize répondants ont été approchés : six Cris de Chisasibi et sept Jamésiens de Radisson. Ils sont des acteurs du développement économique de leur communauté, des entrepreneurs étant en association avec des individus de l'autre communauté ou tout simplement des acteurs travaillant ou ayant déjà travaillé avec des individus de l'autre communauté. 


\section{Dimension 1 : Engagement aux valeurs}

Notre constat est que les acteurs locaux s'approprient de façon compétitive l'esprit de collaboration promu par les discours provinciaux et ce, tant à l'intérieur de chaque communauté qu'entre les deux communautés.

À Radisson, nous observons un engagement compétitif aux valeurs de collaboration : certains Jamésiens acceptent les nouvelles valeurs de collaboration tandis que d'autres préfèrent le statu quo. Par contre, bien que l'engagement soit compétitif, il tend vers l'engagement réformateur, la majorité des répondants étant pour la collaboration. Ces derniers affirment comprendre que la collaboration va aider au développement en créant des emplois et un accès à un plus grand marché.

\section{La perception actuelle des acteurs locaux met en évidence la lourdeur du passé chez les Cris, ce qui amène de la méfiance de leur part face au nouveau contexte.}

À Chisasibi, nous retrouvons aussi un engagement compétitif aux valeurs de collaboration. Les avis sont très partagés. Les acteurs en faveur de la collaboration soulignent qu'elle amène des opportunités pour tous et qu'il y a des avantages à mieux se connaître et à travailler ensemble. Pour leur part, les répondants ambivalents sont plus réservés et méfiants à ce sujet. Ils veulent apprendre à se connaître eux-mêmes d'abord et, par la suite, voir ce qui peut être fait avec les autres. Selon eux, il leur faut plus d'instruction aussi pour être en affaires d'égal à égal en fonction des projets. Finalement, ceux étant contre la collaboration affirment que les Jamésiens ne veulent collaborer que dans leurs propres intérêts et qu'ils ne sont pas respectueux envers les Cris.

Aussi, deux traits majeurs ressortent de nos entretiens sur place : 1) personne ne semble indifférent à cette question et, en même temps, 2) personne n'est entièrement pour ou contre. Premièrement, même si les acteurs locaux sont ambivalents ou qu'ils ne savent qu'en penser, ces valeurs de collaboration sont matière à discussions pour tous. Nous sommes donc certains d'être loin de l'engagement indifférent aux nouvelles valeurs. Deuxièmement, qu'ils soient pour ou contre les valeurs de collaboration, les acteurs locaux y voient des avantages et des inconvénients. Finale- ment, nous voulons souligner que l'appropriation des valeurs par les résidents de la région, que ce soit à Chisasibi ou à Radisson, semble avoir un lien direct, pour la majorité, avec la perception qu'ils ont du changement institutionnel, plus précisément de la signature de la Paix des Braves. En d'autres mots, s'ils sont en accord avec la Paix des Braves, ils sont en accord aussi avec les valeurs de collaboration et se les approprient.

\section{Dimension 2 : Les capacités pour l'action : de nombreuses barrières}

\section{Première capacité : compréhension}

Du point de vue de la première capacité pour l'action, tant les Jamésiens que les Cris semblent avoir compris le nouveau contexte institutionnel: ils comprennent les avantages que la collaboration engendre afin de créer des emplois et de réaliser un développement économique. Par contre, compréhension ne signifie pas nécessairement acceptation. Certains répondants rejettent ce nouveau contexte institutionnel bien qu'ils en comprennent le sens.

\section{Deuxième capacité : fonctionnement}

Les Jamésiens de Radisson et les Cris de Chisasibi semblent encore avoir besoin de renforcer leurs capacités pour fonctionner dans le nouveau contexte institutionnel de collaboration. Les entreprises qui ont pour base une association entre Cris et Jamésiens jusqu'à maintenant n'ont pas été créées sans problème, et la gestion de ces entreprises n'est pas des plus faciles. Plusieurs facteurs sont en cause, les principaux sont : 1) le poids du passé, 2) des différences culturelles, 3) des intérêts divergents et 4) une différence de scolarisation. Les répondants ont identifié ces facteurs comme des barrières à la collaboration, au bon fonctionnement des partenariats et au respect entre les deux nations.

En premier lieu, la perception actuelle des acteurs locaux met en évidence la lourdeur du passé chez les Cris, ce qui amène de la méfiance de leur part face au nouveau contexte. Plusieurs événements ont créé ce poids du passé. D’abord, plusieurs générations de Cris ont été touchées par les pensionnats autochtones entre la fin du $19^{\mathrm{e}}$ siècle et le début des années 1970 , ce qui a été une source de déracinement pour nombre d'entre eux. Ensuite, plus récemment, la communauté de 
Chisasibi a été la plus touchée par le développement hydroélectrique du complexe La Grande depuis le début des années 1970 et par le non-respect par le gouvernement québécois des ententes conclues depuis 1975. De la réticence et de la méfiance se font donc sentir de leur côté, une barrière à surmonter envers les Jamésiens même si ces derniers ne sont pas nécessairement en cause. En un peu plus de 30 ans, depuis l'avènement du complexe La Grande, ce qui représente très peu de temps, les Cris ont dû s'adapter à énormément de changements. Par le fait même, l'histoire des relations entre Jamésiens et Cris est très courte.

Deuxièmement, les différences culturelles sont difficiles à vivre de part et d'autre et représentent, selon les répondants, des obstacles majeurs au succès des partenariats. À quelques reprises, les répondants ont souligné que les Cris et les Jamésiens faisaient partie de deux mondes très différents, ce qui se manifeste par quelques défis dans le quotidien qui nécessitent une adaptation. Deux exemples illustrent les défis de fonctionnement de ces partenariats. Le premier a été mentionné par les Jamésiens. Les Jamésiens interrogés mentionnent «l'appel du bush » des Cris - qui se définit comme l'appel ressenti par ceux-ci pour la taïga en période de chasse - comme étant une difficulté à travailler ensemble. En effet, selon certains répondants, un employé cri qui ne se présente pas au travail le matin parce qu'il s'en va chasser a souvent tendance à le faire sans prévenir. Dans le même esprit, le " goose break »- la période de chasse à l'oie, la bernache du Canada (outarde), lors de la migration de celle-ci - passe avant tout. Les Jamésiens associent ces comportements à la culture fortement ancrée chez les Cris. Ils décrivent les Cris comme étant travaillants, mais travaillants et fiables lorsqu'ils sont au travail, car ils ont un problème de présence au travail et de ponctualité, selon l'expérience des répondants jamésiens. Le second exemple a été mentionné par les Cris. Des répondants cris ont affirmé que les «blancs » étaient arrogants et irrespectueux. De plus, les Cris trouvent que les Jamésiens font tout toujours trop vite, tandis que les Jamésiens trouvent qu'il faut beaucoup de patience lorsqu'on veut entrer en partenariat avec les Cris. Le processus de décision est plus long chez les Cris, celui d'association aussi. En conclusion, bien que les répondants sachent que ces difficultés sont dues à la différence de culture, les moyens pour s'en sortir ne sont pas encore identifiés, sauf peut-être une plus grande connaissance de l'autre.
Le troisième point porte sur la divergence des intérêts entre Jamésiens et Cris. Pour leur part, les Jamésiens souhaitent avoir accès à un marché plus important afin d'augmenter la rentabilité et les profits de leurs entreprises, tandis que les Cris souhaitent avoir plus d'emplois à offrir aux résidents de leur communauté. Deux intérêts qui ne semblent a priori pas être si éloignés l'un de l'autre, pouvant même être complémentaires à première vue, mais qui, sur le terrain, amènent quelques frustrations. Certains Cris ont affirmé que les Jamésiens ne faisaient les choses que dans leur propre intérêt. Un Jamésien, de son côté, le souligne bien : « Il y a du développement qu'on peut faire dans la région qui est essentiel pour les Québécois et qui, pour certains Cris, a l'air superflu ».

Finalement, tant les Cris que les Jamésiens ont mis en avant un manque de formation et d'instruction des Cris comme un obstacle à la création de partenariats d'entreprenariats conjoints et à l'accès aux postes de responsabilités pour les Cris. En ce qui a trait à la constitution de partenariats d'affaires, le manque de formation formelle des Cris opère à deux niveaux. D'abord, tant les Jamésiens que les Cris mettent en avant que les Cris n'ont pas la même facilité d'entrepreneuriat d'affaires que les Jamésiens, en grande partie en raison de leur faible niveau de scolarité qui les prive des aptitudes et de connaissances requises pour démarrer une entreprise. Ce manque de connaissances représente également un obstacle à l'acquisition d'expérience d'entrepreneuriat pour les Cris. Ensuite, cette différence de niveau d'instruction formelle représente une source de méfiance entre individus des deux communautés. Un Cri a affirmé aussi que, parce que les Cris n'ont pas l'instruction nécessaire, ils ont besoin d'assistance lorsqu'ils font affaire avec les Jamésiens, car ces derniers peuvent les tromper : ils ont l'avantage d'avoir une profession et, par le fait même, de mieux comprendre les façons de procéder. Les Cris ont peur de se faire tromper. Ce bas niveau d'instruction amène donc de la méfiance du côté des Cris envers les Jamésiens. En ce qui concerne l'accès à des postes de responsabilités, un répondant jamésien avoue être réticent à donner un poste de gestion à son partenaire cri qui manque de notions d'administration.

\section{Troisième capacité : gestion du changement}

Pour ce qui est de la troisième capacité, nos données suggèrent que tant les Jamésiens que les Cris n'ont 
pas encore acquis les habiletés suffisantes pour gérer le changement vers le nouveau contexte institutionnel. Ils ne se dotent pas, et ne reçoivent pas, des outils et des ressources nécessaires à la gestion du changement. Par ailleurs, les deux communautés et les résidents de ces communautés ne se connaissent pas assez entre eux. Nous avons identifié ces facteurs comme étant des habiletés à gérer le changement vers un nouveau contexte puisque le fait d'avoir les outils et de mieux se connaître aiderait à la gestion de ce changement.

Les répondants des deux communautés ont mentionné le manque de soutien pour la création de liens avec des partenaires d'affaires de l'autre communauté comme un obstacle important à la création de partenariats, tant au plan individuel qu'intercommunautaire. Par exemple, un entrepreneur jamésien ayant un partenaire cri a affirmé avoir dû faire toutes les recherches pour trouver ce partenaire par lui-même. L'absence d'une structure à Radisson susceptible de l'aider à rencontrer un partenaire à Chisasibi augmentait le risque de tomber sur un mauvais partenaire, comme cela lui était déjà arrivé auparavant. En effet, trois répondants cris et trois répondants jamésiens ont mentionné que ni le conseil de bande ni le gouvernement du Québec ne sont en mesure d'aider à la création de relations individuelles ou intercommunautaires au développement socio-économique de Chisasibi et de Radisson. Un répondant jamésien a affirmé qu'à moins d'avoir un changement radical dans l'attitude des élus au conseil de bande vis-à-vis de Radisson, il n'y aurait pas de développement possible au niveau intermunicipal. D'un point de vue plus intercommunautaire, autant les Cris que les Jamésiens affirment, comme il a déjà été mentionné, qu'ils ne se connaissent pas beaucoup entre eux. Un Cri, en particulier, pense qu'il faudrait que les Jamésiens et les Cris s'assoient plus souvent ensemble afin de voir et de comprendre les intérêts de chacun. En effet, très peu de résidents semblent se connaître entre les deux communautés. Comme l'affirme ici un répondant cri : «I'm sure there are people there [Radisson] that are still there for twenty years and they don't even know anybody here [Chisasibi] ». Nous pouvons donc avancer ici que «deux solitudes » se côtoient dans la même région.

En résumé, à partir des résultats de notre étude, les résidents de Chisasibi et de Radisson ne semblent pas encore détenir les trois capacités pour l'action. Tant les Cris que les Jamésiens paraissent comprendre le nouveau contexte institutionnel; par contre, ils n'ont pas les habiletés et les compétences requises pour fonctionner dans ce nouveau contexte institutionnel. Les obstacles principaux mis en évidence par notre étude portent sur un lourd passé créant de la méfiance chez les Cris, un grand fossé entre les deux cultures, une instruction plus restreinte chez les Cris, une assistance d'accompagnement pratiquement absente de la part du gouvernement et d'organismes et, surtout, le fait que les Cris et les Jamésiens de Chisasibi et de Radisson ne se connaissent pratiquement pas. Toutes ces barrières expliquent pourquoi les acteurs locaux n'ont pas les capacités pour l'action.

\section{Conclusions et piste d'amélioration}

Nous avons présenté ci-dessus les résultats de notre étude sur la mise en œuvre d'un discours politique et économique qui promeut la collaboration entre les deux communautés dans sa phase initiale de mise en œuvre. Nous ne prétendons pas généraliser cette étude à l'ensemble de la région de la Baie-James et ce, pour plusieurs raisons. D'abord, cette recherche est de nature exploratoire. Nous l'avons réalisée par entrevues et observations sur le terrain et ne prétendons pas brosser un portrait général des deux communautés étudiées. Ensuite, nos résultats portent sur deux localités uniquement dont le contexte est bien différent de celui des autres communautés sur le territoire. Comme il a déjà été mentionné, Chisasibi est la communauté crie ayant été la plus touchée par le développement hydroélectrique du complexe La Grande et, de plus, elle est la seule à avoir voté contre la Paix des Braves à l'origine.

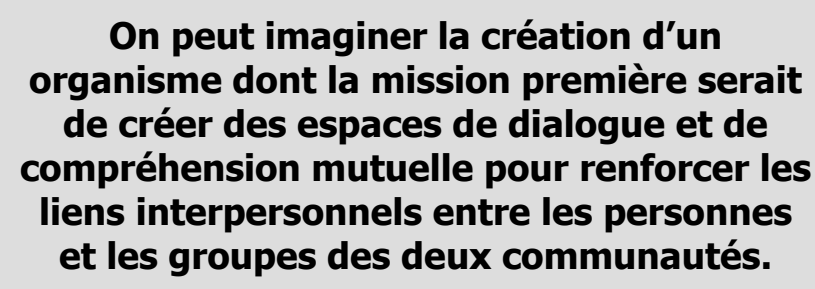

On peut imaginer la création d'un organisme dont la mission première serait de créer des espaces de dialogue et de compréhension mutuelle pour renforcer les liens interpersonnels entre les personnes et les groupes des deux communautés.

Nous avons observé un contraste fort entre la formulation de ces discours - au niveau provincial ou « de nation à nation »- et les dynamiques locales dans les localités de Chisasibi et Radisson. L'esprit de collaboration et de partenariat promu par la Paix des Braves et la Politique de développement du Nord-du-Québec ne s'observe qu'au plan d'un engagement compétitif 
aux valeurs. Les acteurs locaux reconnaissent le nouveau contexte et le potentiel de développement créé par la collaboration, mais, trois ans après l'officialisation de la Paix des Braves, cette reconnaissance ne se traduit ni par une collaboration entre communautés ni par des partenariats d'affaires entre individus des deux communautés. Capacités présentes de façon différenciée entre les deux communautés, ce qui se reflète et se répercute dans les partenariats d'affaires entre personnes de communautés différentes.

Notre étude suggère qu'il existe un chaînon manquant dans cette politique. Même si la politique «de nation à nation » vise à promouvoir la collaboration entre les deux communautés afin de développer les partenariats d'affaires, les répondants de Chisasibi et de Radisson perçoivent un manque de liens entre les deux communautés. Pour pallier ce manque, on peut imaginer la création d'un organisme dont la mission première serait de créer des espaces de dialogue et de compréhension mutuelle pour renforcer les liens interpersonnels entre les personnes et les groupes des deux communautés. Est apparue constamment dans cette étude la nécessité de dépasser les « deux solitudes » de cette région et de créer un espace de dialogue afin de mieux se comprendre. Un effet de cette meilleure compréhension pourrait être la création de la confiance et de la connaissance de l'autre qui sont nécessaires à la création de partenariats et de réseaux. Au niveau local, les Cris comme les Jamésiens affirment qu'il est nécessaire de se connaître davantage. C'est sur cette base que le développement et la collaboration auront de l'avenir et permettront à la région de réaliser son plein potentiel.

\section{Notes et références}

1 Gouvernement du Québec et Grand Conseil des Cris (2002). Entente concernant une nouvelle relation entre le Gouvernement du Québec et le Grand Conseil des Cris du Québec, p. 1.
2 Gouvernement du Québec (2002). Entente Québec-Cris : la paix des braves [Dépliant].

3 Greenwood, Royston et Hinings, C.R. (1996), «Understanding Radical Organizational Change : Bringing Together the Old and the New Institutionalism ", Academy of Management Review, vol. 21, $\mathrm{n}^{\circ} 4$.

4 Statistique Canada (2001), http://www12.statcan.ca/ francais/profil01ab/PlaceSearchForm1_F.cfm.

5 Idem.

6 Mercier, G. et G. Ritchot (1997). «La Baie James : les dessous d'une rencontre que la bureaucratie n'avait pas prévue ", Les Cahiers de géographie du Québec, vol. 41, $\mathrm{n}^{\mathrm{o}} 113$, septembre, p. 137-169.

7 Gourdeau, É. (2002b). «Synthèse de la Convention de la Baie-James et du Nord québécois », dans A.-G. Gagnon et G. Rocher, Regard sur la Convention de la Baie-James et du Nord québécois, Montréal, Éditions Québec Amérique Inc., p. 25 à 38 .

8 Gouvernement du Québec (1991). La Convention de la Baie James et du Nord québécois et conventions complémentaires, Secrétariat aux affaires autochtones, Québec, Publications du Québec, p. 6.

9 Gourdeau, É. (2002c). «L’État québécois et la mise en œuvre de la Convention de la Baie-James et du nord québécois ", dans A.-G. Gagnon et G. Rocher, Regard sur la Convention de la Baie-James et du Nord québécois, Montréal, Éditions Québec Amérique Inc., p. 216.

10 La phase 2 des travaux du complexe La Grande regroupe la construction des centrales La Grande-1, La Grande-2-A, Laforge-1, Laforge-2 et Brisay, en plus de certaines infrastructures comme des lignes, un agrandissement du poste Radisson et la construction du poste convertisseur. La phase 2 s'est étendue de 1987 à 1996.

11 Localité de Radisson (1995). Plan stratégique de développement de la localité de Radisson 1995-2000.

12 Greenwood, R. et C.R. Hinings, (1996), op. cit., p. 10221054. 


\section{Publicité}

CRDT 